\title{
La secuencia didáctica, de enfoque comunicativo, como propuesta pertinente para la enseñanza del lenguaje y la formación docente: Caso de dos instituciones educativas del Distrito Turístico de Riohacha en el
}

\author{
Departamento de La Guajira - Colombia ${ }^{1}$
}

The didactic sequence as a relevant proposal for the teaching of language and teacher training: Case of two educational institutions of the Tourist District of

Riohacha in the Department of La Guajira - Colombia

Francisco Javier Blanchar Añez ${ }^{2}$

Piedad Julieta Sierra Marriaga ${ }^{3}$

Blanchar A, Francisco. Sierra M, Piedad
miradas N²-2019 ISSN digital No 2539-3812 Págs $82-102$
Recepción: Enero 22 de 2019
Aprobación: Mayo 26 de 2019
Publicación: Junio 29 de 2019

\section{Resumen}

El artículo presenta los resultados de la investigación enmarcada en la implementación de una secuencia didáctica para la producción de textos narrativos, tipo crónicas, con estudiantes de $2^{\circ}$ de Educación Básica Primaria (EBP) de dos instituciones educativas del Distrito Turístico de Riohachal; la cual hace parte del Macroproyecto de Didáctica del lenguaje escrito de la Maestría en Educación de la Universidad Tecnológica de Pereira. El trabajo estuvo orientado a determinar la incidencia de una secuencia didáctica, de enfoque comunicativo, en la producción escrita textos narrativos, tipo crónica, y reflexionar sobre las prácticas de enseñanza del lenguaje a partir de su implementación. El estudio fue de naturaleza cuantitativa, con un complemento cualitativo, de diseño cuasi-experimental,

\footnotetext{
1 Artículo derivado de la tesis del programa de maestría en educación de la Universidad Tecnológica de Pereira en convenio con la Universidad de La Guajira, titulado "El contexto visto desde mis ojos: una secuencia didáctica para la producción de textos narrativos, tipo crónicas, con estudiantes de $2^{\circ}$ de EBP de las I.E. San Juan Bautista y Luis Antonio Robles del Distrito Turístico de Riohacha. Esta investigación hace parte del macroproyecto de la línea de investigación en didáctica del lenguaje de la Universidad Tecnológica de Pereira.

2 Ingeniero del Medio Ambiente, Magister en Educación de la Universidad Tecnológica de Pereira, Docente Tutor del Programa Todos a Aprender PTA del Ministerio de Educación Nacional de Colombia en lainstitución educativa Luis Antonio Robles del Distrito de Riohacha. franciscoblanchar@hotmail.com; fblanchar@gmail.com.

3 Psicóloga, Magister en Educación de la Universidad Tecnológica de Pereira, Docente de educación básica primaria de la institución educativa San Juan Bautista del Distrito de Riohacha. pjsierra76@gmail.com.
} 
intragrupo de tipo Pre-Test/ Pos-Test. Se utilizaron dos instrumentos: una rejilla de evaluación, aplicada antes y después de la implementación de la secuencia didáctica y un diario de campo. Los resultados permitieron validar la hipótesis de trabajo en ambos grupos, estableciendo que una secuencia didáctica de enfoque comunicativo mejoró significativamente la producción textual de textos tipo crónica de los estudiantes participantes. Respecto a las prácticas de enseñanza, se logró generar procesos reflexivos en los docentes participantes, en torno a su actuar permanente y futuro en las aulas.

Palabras Claves: Producción textual, secuencia didáctica, género narrativo, crónica, práctica reflexiva.

\section{Abstract}

The article presents the results of the research framed in the implementation of a didactic sequence for the production of narrative texts, chronic type, with students of $2^{\circ}$ of EBP of two educational institutions of the Tourist District of Riohachal; which is part of the Macroproject of Didactics of the written language of the Master in Education of the Technological University of Pereira. The work was oriented to determine the incidence of a didactic sequence, of communicative approach, in the written production narrative texts, chronic type, and to reflect on the practices of language teaching from its implementation. The study was of a quantitative nature, with a qualitative complement, of quasiexperimental design, intragroup of type Pre-Test / Post-Test. Two instruments were used: an evaluation grid, applied before and after the implementation of the didactic sequence and a field diary. The results allowed to validate the working hypothesis in both groups, establishing that a didactic sequence of communicative approach significantly improved the textual production of chronic type texts of the participating students. Regarding the teaching practices, it was possible to generate reflexive processes in the participating teachers, around their permanent and future actions in the classrooms.

Keywords: Textual production, didactic sequence, narrative genre, chronicle, reflexive practice.

\section{Introducción}

El lenguaje, desde la perspectiva vigostskyana, se reconoce como una de las capacidades que más ha marcado el curso evolutivo de la especie humana; pues le ha permitido no solo separarse del mundo físico para nominarlo, si no también y fundamentalmente para comunicarse con otros (MEN, 2006).

Dadas la relevante potencialidad del lenguaje para la vida de los seres humanos, el escenario privilegiado para concretar sus funciones $\mathrm{e}$ intencionalidades sociales es la escuela, lugar en el cual, como lo plantean diversos investigadores (Lerner, 2001; Pérez y Rincón, 2009; Jolibert, 2002, entre otros) debe propiciar el desarrollo de competencias comunicativas, necesarias para participar con éxito en el contexto socio-cultural y académico, en el cual los sujetos se encuentren inmersos. 
Sin embargo, actualmente las prácticas de enseñanza de la producción textual difieren de los avances en la didáctica, propuestos por investigadores $\mathrm{y}$ especialistas en la materia. En este sentido autores como Castaño (2014) y Sánchez (2014) manifiestan que en muchos casos son las creencias y las percepciones personales social y culturalmente arraigadas, las que guían mayoritariamente las decisiones $\mathrm{y}$ actuaciones en el aula de muchos docentes, las cuales se construyeron desde sus experiencias en la escuela y sobre el estudio de la didáctica del lenguaje escrito.

Este tipo de prácticas, como afirma Castaño (2014), se ven reflejadas también en los deficientes resultados obtenidos por los estudiantes colombianos de básica primaria y secundaria en los indicadores del área de lenguaje en las pruebas evaluativas censales.

Resultados evaluativos a nivel nacional, como las pruebas SABER-ICFES de los años 2015 y 2016 se evidencian reiteradas dificultades en los procesos de comprensión y producción textual de los estudiantes de Colombia. Se evidencia que el $51 \%$ de los estudiantes del grado $3^{\circ}$ y el $61 \%$ de los estudiantes del grado $5^{\circ}$ se ubicaron en los niveles de menor desempeño (insuficiente y mínimo); es decir, no solo evidenciaron capacidades limitadas para ubicar información dentro del texto, solo a partir de marcas textuales evidentes o fácilmente identificables, sino también para establecer relaciones temporales entre eventos del texto cuando están ordenados dentro de la misma linealidad del escrito ICFES (2017).
En relación a la situación descrita, las instituciones educativas San Juan Bautista (INSAJUBA), ubicada en el Corregimiento de Cotoprix, y la institución Luis Antonio Robles (LAR), ubicada en el corregimiento de Camarones, ambas pertenecientes al Distrito Turístico de Riohacha, en el Departamento de la Guajira, no son ajenas a esta problemática, teniendo en cuenta que los resultados evidenciados en las diversas aplicaciones anuales de las pruebas censales de lenguaje SABER ICFES (2009, 2012, 2013, 2014, 2015 y 2016) indican que en promedio entre el $79 \%$ y $80 \%$ de los estudiantes del grado $3^{\circ}$ de estas instituciones se ubicaron en los niveles inferiores de la escala de calificación (insuficiente y mínimo), mientras que para el grado $5^{\circ}$ fueron del $80 \%$ y $95 \%$, respectivamente.

Con el fin de contribuir a la solución de la problemática descrita se hace necesario promover el diseño e implementación de propuestas didácticas innovadoras y pertinentes, que puedan partir del contexto, basadas en referentes y antecedentes teóricos relevantes relacionados con la enseñanza y el aprendizaje del lenguaje, para la producción escrita. Estas propuestas, tal como lo plantea la UNESCO (1997), deben basarse en la enseñanza y el aprendizaje del lenguaje desde temprana edad, para propiciar el desarrollo de capacidades y la formación de comunidades de lectores y escritores, que puedan leer y comprender textos escritos y además producir textos de diversa naturaleza, pues esta función es una tarea indelegable y principal de la escuela. 
En este marco de ideas, el presente artículo presenta los resultados de una investigación basada en la implementación de una secuencia didáctica de enfoque comunicativo para la enseñanza de la producción textual de textos narrativos, tipo crónicas, con estudiantes de $2^{\circ}$ de educación básica primaria de dos instituciones de carácter oficial del Distrito Turístico de Riohacha, pretendiendo fortalecer la capacidad de producción escrita de los estudiantes y las prácticas de enseñanza de los docentes de lenguaje participantes.

Lo expuesto tuvo como punto de partida los siguientes interrogantes de investigación: ¿Cuál es la incidencia de una secuencia didáctica de enfoque comunicativo en la producción de textos narrativos de estudiantes de grado $2^{\circ}$ de EBP de las instituciones educativas San Juan Bautista y Luis Antonio Robles del Distrito Turístico de Riohacha? y ¿Qué reflexiones se generan acerca de la enseñanza del lenguaje a partir de la implementación de la secuencia didáctica en estas instituciones educativas?

\section{Marco de referencia}

La investigación se sustentó en el concepto de lenguaje como práctica social y su manifestación a través de la producción textual, relacionando los principales modelos de producción textual y el enfoque comunicativo, orientados a la escritura del texto narrativo y tipo d escrito: crónica, bajo la conceptualización de las secuencias didácticas y finalmente las características de las prácticas pedagógicas reflexivas.

\section{EI lenguaje y lenguaje escrito}

La relación de los sujetos con su entorno esta mediada por el lenguaje, una de las capacidades más relevantes, a través de la cual pueden interactuar con el mundo e interpretarlo, en la medida que lo organizan y lo representan. Desde esta perspectiva, se puede acceder al conocimiento, crearlo, transformarlo, como producto de la interacción generada con su realidad física y social.

Vygotsky (1989) explica la trascendencia del lenguaje en este proceso evolutivo, desde sus dos funciones; cognitiva y comunicativa. La primera de ellas, referida al conocimiento y representación de la realidad humana, producto de la estrecha relación entre pensamiento y lenguaje, permite la manifestación de las características particulares de cada individuo y la construcción cultural, la representación de ideas y conceptos, y de esta manera facilita el establecimiento de convenciones en los distintos grupos sociales.

Específicamente en relación con la escritura, Teberosky (1990) y Tolchinsky (1993) la definen como el acto de producir ideas genuinas y configurarlas en un texto, obedeciendo a unas reglas sociales de circulación; proceso que requiere la puesta en juego de habilidades cognitivas complejas que impliquen satisfacer los intereses de la situación de comunicación en las que se encuentre inmerso. En palabras de Cassany (1999) "escribir se puede asimilar al desarrollo de actividades tan complejas como la construcción de una infraestructura o llevar la 
contabilidad de una empresa o diseñar una coreografía" (p.12).

Sin embargo, esta perspectiva contemporánea del lenguaje escrito ha sido el producto de la evolución de las diversas maneras de entenderlo y la forma de orientar su enseñanza. Autores como Caro, Uribe y Camargo (2009) presentan una síntesis de tres modelos que explican la naturaleza, características y propiedades del proceso de producción textual.

\section{Modelos de producción textual}

En primer lugar, están los modelos de producto, los cuales se centran en el desarrollo de la capacidad de producción textual desde una perspectiva fundamentalmente lingüística, comprendida desde las dimensiones micro-estructural, que supone el dominio de los aspectos formales del texto (reglas gramaticales, ortografía) y macro-estructural (que implica el aprendizaje y utilización de las distintas tipologías textuales). Esta distinción generó a su vez dos enfoques de enseñanza: el modelo oracional o el enfoque de la enseñanza de la gramática (dominio de la sintaxis, el léxico, la morfología y la ortografía) y el modelo textual /discursivo o enfoque basado en las funciones.

En segundo lugar, los modelos de proceso, caracterizados por el análisis de los procesos básicos u operaciones implícitas en el acto de la escritura. Dentro de esta categoría se encuentran los modelos de traducción, por etapas y cognitivos.
Entre los modelos por proceso, y para efectos de la presente investigación, se explican los modelos planteados por Hayes y Flower (1980) y Scardamalia y Bereiter (1992), que avanzan los anteriores, pues introducen el contexto de la escritura y su finalidad.

Finalmente, los modelos contextuales o ecológicos establecen la composición escrita como un proceso mediatizado no solamente por variables de índole personal, como en los modelos cognitivos, sino también por el contexto sociocultural en el que se desarrolla.

\section{Los enfoques comunicativos}

Los enfoques comunicativos constituyen un conjunto de propuestas de enseñanza de la lengua en el que se incluyen diversas metodologías, cuyo propósito central es el uso del lenguaje en situaciones reales de comunicación, las cuales le brindan a los sujetos la oportunidad de aprender y comunicarse desde el aula de clase hasta situaciones de formalidad discursiva orales y escritas.

Parte de la consolidación de la didáctica de la lengua a través de los enfoques comunicativos se le atribuye al trabajo de Hymes (1972), y su propuesta para el desarrollo de la competencia comunicativa, definida como la capacidad de elaborar enunciados teniendo en cuenta no sólo que su significado y su gramaticalidad sean correctos, sino que también sean socialmente apropiados de acuerdo con las intenciones de los sujetos que se comunican y el contexto en que se encuentran inmersos, concepto 
que resulta mucho más amplio que la tradicional competencia lingüística o gramatical establecida por Chomsky (1957), la cual alude a la capacidad innata de un hablante $y$ oyente ideal para emitir y comprender un número ilimitado de oraciones en una comunidad de habla homogénea, bajo la suposición de poder desarrollarse de manera satisfactoria en cualquier contexto comunicativo en el que pueda participar.

\section{Propuesta de Jolibert para la producción textual}

Teniendo en cuenta los propósitos e intereses de la investigación y la naturaleza de la enseñanza y el aprendizaje desde el enfoque comunicativo, la propuesta de Jolibert (2002), enmarcada en el trabajo por proyectos, plantea que el desarrollo de actividades de enseñanza y aprendizaje activas representan estrategias pedagógicas coherentes y eficaces que promueven en los niños y niñas la producción y comprensión de textos variados, y su adaptación a situaciones reales lo más personalizado posible, además de permitirles la posibilidad de aprender a elaborarlos, de tal manera que posteriormente sean capaces de producirlos solos, sin tutelas y adaptándolos a las múltiples situaciones en las que se encuentren inmersos.

Se trata que los niños puedan desarrollar una estrategia de producción de textos apoyada, entre otros aspectos, en habilidades complementarias como una capacidad de representación de la situación de comunicación y del tipo de texto que se desea producir, competencias para seleccionar de un abanico conocido de diferentes tipos de texto, aquel que mejor le conviene a la situación de comunicación y una aptitud para administrar globalmente la actividad de producción, en la medida en que se logre aprender a implementar diferentes niveles de análisis de un texto, basados en la identificación $y$ uso funcional de informaciones o índices que da un texto a partir de sus estructuras lingüísticas.

\section{El texto narrativo}

La narración constituye una de las habilidades primarias de los seres humanos, la cual, en forma de relato, convive con ellos todo el tiempo. De hecho, la mayoría de seres humanos inician sus primeros encuentros con los relatos en la infancia, a través de los cuentos de hadas, cuentos populares y las anécdotas de familia, los cuales permiten el acercamiento a la cultura, los valores, maneras de pensar y actuar, propias de cada comunidad.

En este marco, difícilmente existe un tema que no pueda ser abordado de forma narrativa dadas las diferentes formas del relato que circulan en la esfera humana y que sirven de apoyo para su existencia. De acuerdo con Barthes (1985), el relato puede manifestarse en variadas formas: en el mito, la leyenda, el cuento, la novela, la epopeya, la historia, la tragedia, el drama, la comedia, las noticias policiales, la conversación.

La trama narrativa ofrece todo tipo posibilidades de expresión representadas en las variadas prácticas 
humanas, clasificadas por Bautista y Cortés (1998) como relatos literarios en el caso del cuento y la novela y no literarios refiriéndose a la nota periodística, la crónica, ente otros. Además, los autores plantean la existencia de relatos narrativos como la carta y no narrativos como las corridas de toros, los rituales religiosos; es decir aquellas manifestaciones narrativas de tipo no verbal.

\section{La crónica}

La crónica representa un tipo de texto narrativo en el que, aunque no se considera una narrativa pura, se plasma la subjetividad del autor propia de este género discursivo, cuando quien escribe retoma, renueva, interpreta, llena de detalles y recrea un acontecimiento en la narración, bajo el influjo de su punto de vista. En este sentido, el autor puede aportar un estilo personal que embellezca la escritura.

De acuerdo con Ortiz (2010) y Salcedo (2012), las crónicas permiten la narración de los hechos según el orden temporal en que ocurrieron. Consisten entonces, en la exposición de acontecimientos con la peculiaridad de la introducción de elementos de valoración e interpretación por parte del cronista. Con relación a su estructura como texto narrativo, plantean que una buena crónica se compone por los siguientes elementos: (a) el título, sea una palabra o frase, hace alusión de manera breve y llamativa a los hechos narrados en el texto, (b) la presentación, entendida como el conjunto de circunstancias mediante las cuales se introducen los personajes o los hechos desde la perspectiva del cronista y cuyo propósito es generar interés en el lector; teniendo en cuenta que posee un carácter introductorio al texto, en el cual los hechos narrados en ella permitan inferir el posible contexto o conocimiento cultural, social e histórico de la historia, (c) la progresión cronológica, que permita identificar en los sucesos narrados la reconstrucción de mundos y universos culturales y sociales, resaltando la importancia del espacio y tiempo y (d) el cierre, como apartado que incluye la descripción de un estado final coherente con el resto de la historia.

\section{La secuencia didáctica}

La propuesta de enseñanza y aprendizaje para el trabajo de producción textual de la investigación se basa en la Secuencia Didáctica, la cual constituye una configuración posible para el diseño didáctico de prácticas pedagógicas del lenguaje. Esta secuencia se sustenta en los planteamientos de Camps, Cotteron, Dolz, Farrera, Fort, Guasch, Martinez, Milian, Ribas, Rodríguez, Santamaria, Utset, Vila y Zayas (2003), quienes fundamentados en el trabajo por proyectos de lengua, la definen como una unidad de enseñanza de la composición, en la cual se relacionan actividades y objetivos globales para el alcance de los propósitos de enseñanza del lenguaje. Estos autores establecen una serie de características para la secuencia didáctica, que la determinan como una opción pertinente para la enseñanza, entre las que se destacan las siguientes: 
- Se formula como un proyecto de trabajo que tiene como objetivo la producción de un texto (oral, escrito), y que se desarrollará durante un determinado periodo de tiempo más o menos largo según convenga.

- La producción del texto, base del proyecto, forma parte de una situación discursiva que le dará sentido, partiendo de la base de que texto y contexto son inseparables.

- Se plantean unos objetivos de enseñanza y de aprendizaje delimitados que han de ser explícitos para los alumnos. Estos objetivos se convertirán en los criterios de evaluación.

- Los alumnos involucrados en el trabajo con la Secuencia Didáctica llevan a cabo la actividad global a partir de los conocimientos que ya tienen y la atención didáctica se orienta hacia nuevos objetivos de aprendizaje.

- El esquema general de desarrollo de la secuencia tiene tres fases: preparación, producción y evaluación (Camps et al., 2003, p. 34).

\section{Prácticas pedagógicas reflexivas}

La práctica pedagógica se relaciona con las actividades de planificación, desarrollo y valoración de situaciones de enseñanza orientadas al aprendizaje, con el fin de brindar escenarios propicios para la construcción de saberes por parte de los aprendices. Autores como Pérez, Camacho y Rodriguez (2013) coinciden en afirmar que la práctica pedagógica representa el resultado de un hacer reflexivo, que se razona y que produce conocimiento, gracias a la unión teoría-práctica. De otra parte, Zuluaga (1984) la propone como "Una noción metodológica de aquello que acontece en el salón de clase, de lo que hace cotidianamente el maestro, y como noción discursiva; que está constituida por el triángulo institución (escuela), sujeto (el docente) y discurso (saber pedagógico)" (p. 35).

Ahora bien, el concepto de prácticas reflexivas aparece con los trabajos de Schön (1987) y es profundizado por Perrenoud (2011), quienes vinculan la actividad docente a la necesidad de la actividad reflexiva en el campo educativo, planteando que un profesional de la educación debe ser capaz de saber cuál es su cometido, sin estar estrictamente constreñido por las reglas, las directivas, los modelos, los programas, los horarios o los procedimientos normalizados. Es decir, un docente debe constituirse en un profesional que sabe lo que tiene que hacer y cómo hacerlo de la mejor forma posible.

\section{Metodología}

La investigación se caracterizó por su naturaleza cuantitativa, que de acuerdo a Imbernón (2012), se basa en la manipulación deliberada de las variables mediante la provocación del fenómeno por parte del investigador con la finalidad de observarlo y medirlo, de tal manera que se inscribe en un modelo causal, el presente estudio se enmarca en este modelo porque busca identificar 
la incidencia de la implementación de una secuencia didáctica de enfoque comunicativo en la producción textual de crónicas de estudiantes de grado $2^{\circ}$ de EBP de las IE San Juan Bautista y Luis Antonio Robles del distrito turístico de Riohacha

De manera complementaria se desarrolla un análisis cualitativo, sustentado en el estudio reflexivo de la práctica pedagógica de los docentes participantes, durante la implementación de la secuencia didáctica.

El diseño de la investigación es cuasiexperimental, intragrupo, tipo Pre-TestPos-Test. De acuerdo con Hernández, Fernández y Baptista (2010) este tipo de diseños permiten la manipulación deliberada de al menos una variable independiente para observar su efecto y relación con una o más variables dependientes.

La población estuvo representada por todos los estudiantes del grado $2^{\circ}$ de las instituciones educativas oficiales del área rural del Distrito Turístico de Riohacha y la muestra seleccionada la constituyeron 33 estudiantes del grado $2^{\circ}$ de ambas instituciones educativas (15 de la IE Luis Antonio Robles y 18 de la IE San Juan Bautista).

Entre las principales características de los estudiantes de la muestra se encuentran que su promedio de edad oscila entre los 6 y 8 años, mayoritariamente habitantes de zonas rurales de estratos 1 y 2 , con desempeño académico insuficiente, mínimo $\mathrm{y}$ satisfactorio en el área de lenguaje de acuerdo con el Sistema Institucional de Evaluación de los Estudiantes (SIEE) de cada institución.

\section{Resultados}

Los resultados abarcan dos análisis: el primero, cuantitativo, relacionado con los aprendizajes de los estudiantes y el segundo, el análisis cualitativo de las prácticas de enseñanza de los docentes participantes, a partir del diario de campo, las cuales fueron desarrolladas durante la implementación de la S.D., en sus tres momentos: preparación, desarrollo y cierre.

\section{Análisis cuantitativo de la producción textual de crónicas}

En las tablas 6 y 7 se presentan los resultados obtenidos a partir del uso de estadística inferencial, mediante el uso de la prueba $t$-Student con un índice de significancia del $0,05 \%$ para muestras emparejadas, aplicada a ambos grupos de estudio, con el fin de aceptar o rechazar la hipótesis.

Tabla 7. Resultados de la prueba t-student del grupo 1

\begin{tabular}{|c|c|c|}
\hline & Total Pre-Test & Total Pos-Test \\
\hline Media & 16,53333333 & 24,2 \\
\hline Varianza & 5,40952381 & 14,02857143 \\
\hline Observaciones & 15 & 15 \\
\hline Coeficiente de correlación de Pearson & 0,724833049 & \\
\hline Diferencia hipotética de las medias & 0 & \\
\hline Grados de libertad & 14 & \\
\hline Estadístico t & $-11,37873123$ & \\
\hline $\mathrm{P}(\mathrm{T}<=\mathrm{t})$ una cola & 9,22467E-09 & \\
\hline Valor crítico de $\mathrm{t}$ (una cola) & 1,761310136 & \\
\hline$P(T<=t)$ dos colas & $1,84493 \mathrm{E}-08$ & \\
\hline Valor crítico de $t$ (dos colas) & 2,144786688 & \\
\hline
\end{tabular}


La secuencia didáctica, de enfoque comunicativo, como propuesta pertinente para la enseñanza del lenguaje y la formación docente

Tabla 8. Resultados de la prueba t-student del grupo 2

\begin{tabular}{lrr}
\hline & Total Pre-Test & Total Pos-Test \\
\hline Media & 12,444444444 & 23,66666667 \\
Varianza & 0,496732026 & 29,76470588 \\
Observaciones & 18 & 18 \\
Coeficiente de correlación de Pearson & 0,239671174 \\
Diferencia hipotética de las medias & 0 \\
Grados de libertad & \multicolumn{2}{c}{17} \\
Estadístico $t$ & $-8,931328527$ \\
$P(T<=t)$ una cola & $3,94976 \mathrm{E}-08$ \\
Valor crítico de $t$ (una cola) & 1,739606726 \\
$\mathrm{P}(T<=t)$ dos colas & $7,89951 \mathrm{E}-08$ \\
Valor crítico de $t$ (dos colas) & 2,109815578 \\
\hline
\end{tabular}

De acuerdo con los resultados presentados en las tablas anteriores, específicamente el relacionado con el valor del indicador $\mathrm{P}(\mathrm{T}<=\mathrm{t})$ para dos colas, obtenido en cada uno de los grupos $(0,0000000184$ para el grupo 1 y de 0,0000000789 para el grupo 2), se evidencia que ambos se encuentran ubicados dentro del rango de aceptación, al ser menores que el índice de significancia establecido $(0,05)$. A partir de estos resultados, se puede afirmar que se generaron cambios significativos en la producción de textos narrativos. Teniendo en cuenta esta conclusión se rechaza la hipótesis nula $\mathrm{H}_{\mathrm{o}}$ y se acepta la hipótesis de trabajo $\mathrm{H}_{1}$, con lo cual se puede afirmar, con un nivel de confianza del 95\%, que la secuencia didáctica de enfoque comunicativo mejoró significativamente la producción de textos narrativos, tipo crónica, en los estudiantes de segundo grado de educación básica primaria, de las instituciones educativas San Juan Bautista y Luis Antonio Robles del Distrito Turístico de Riohacha, en el Departamento de La Guajira.

En gráficas 1 y 2, se presenta un comparativo general de los resultados del Pre-Test y el Pos-Test que dan cuenta de la movilidad de los estudiantes de ambos grupos por los niveles de desempeño previamente establecidos: bajo, medio y alto.

\section{Gráfica 1. Comparación Pre-Test vs. Pos-} Test Grupo 1

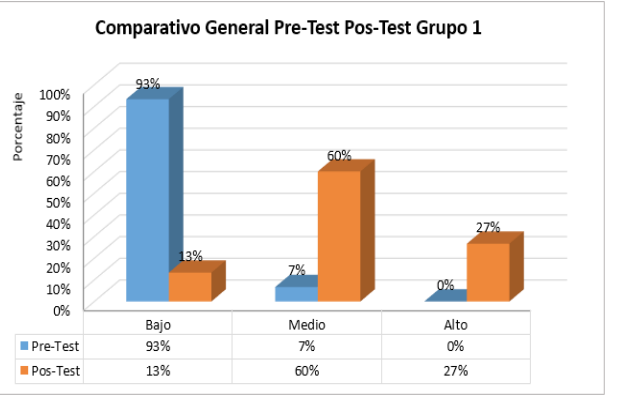

Gráfica 2. Comparación Pre-Test vs. PosTest Grupo 2

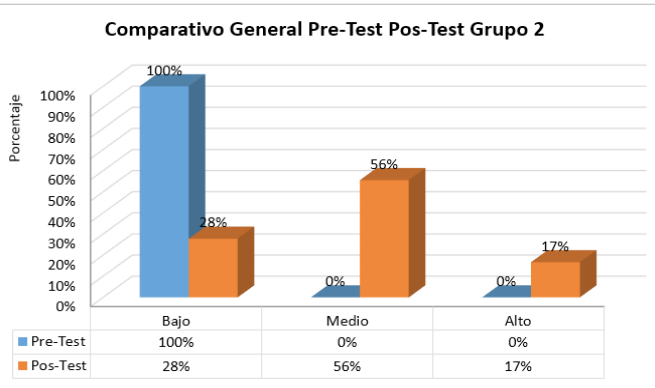

De acuerdo con la información de las gráficas 1 y 2 , se evidencia que en ambos grupos los estudiantes se movilizaron del nivel bajo, en el cual se encontraba la mayoría inicialmente $(93 \%$ en el caso del grupo 1 y la totalidad del grupo 2), hasta los niveles medio y alto $(60 \% \mathrm{y}$ $25 \%$, en el grupo 1 y $56 \%$ y $17 \%$ en el grupo 2, respectivamente); lo cual confirma los avances de los estudiantes en la producción de crónicas.

En las gráficas mostradas a continuación se presentan los niveles de desempeños de las dimensiones del trabajo en el Pre-Test y el Pos-Test. 


\section{Gráfica 5. Comparativo Situación de} Comunicación Grupo 1

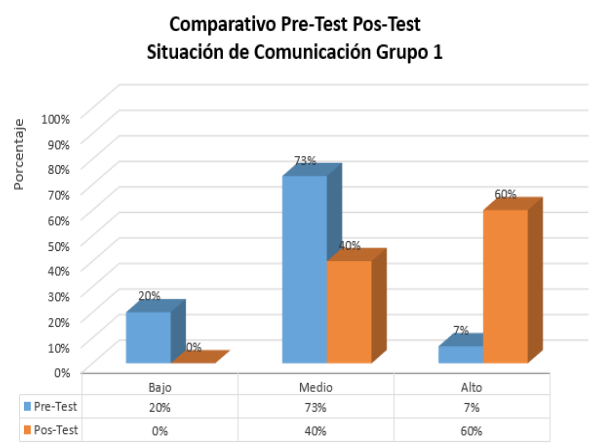

Gráfica 6. Comparativo Situación de Comunicación Grupo 2

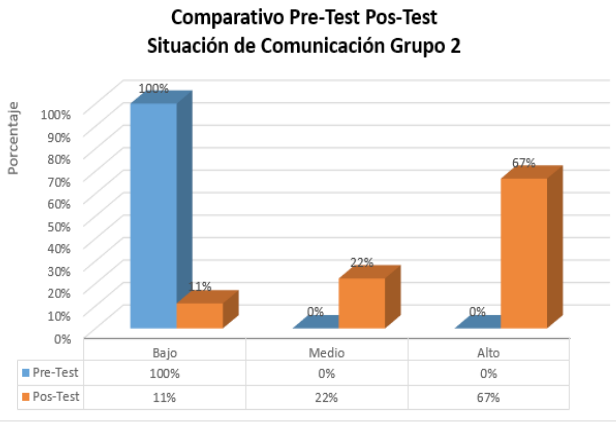

Las gráficas 5 y 6 muestran resultados disimiles para los dos grupos. Así, en el grupo 1, la mayoría de estudiantes se ubican en el Pre-Test en el nivel medio (73\%) y algunos en el nivel bajo (20\%), situación que se modifica en el Pos-Test, pues en su mayoría pasan a los niveles alto $(60 \%)$ y medio $(40 \%)$. Ahora bien, en el grupo 2 durante el Pre-Test la totalidad de los estudiantes se ubican en el nivel bajo (100\%), para moverse mayoritariamente en el Pos-Test, a los niveles medio $(22 \%)$ y alto $(67 \%)$.

En las gráficas 9 y 10 se presentan los resultados para la dimensión superestructura.
Gráfica 9. Comparativo Superestructura Grupo 1

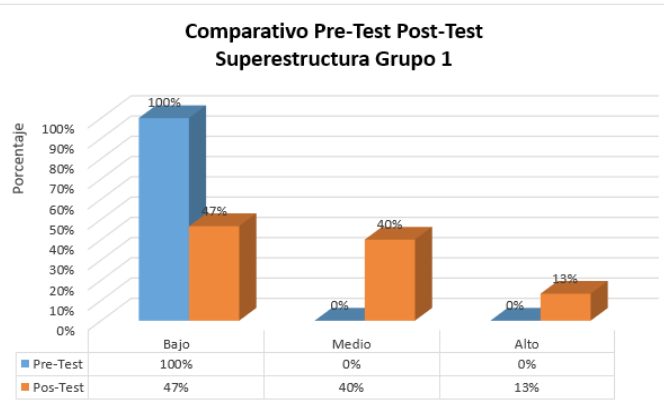

Gráfica 10. Comparativo Superestructura Grupo 2

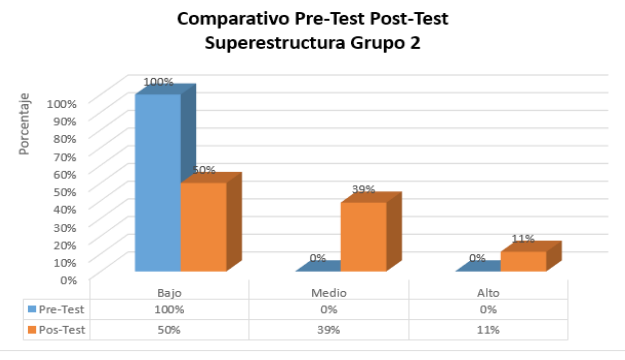

En las gráficas 9 y 10 se evidencia que ambos grupos presentaron el mismo comportamiento en torno a la dimensión Superestructura, dado que un porcentaje de los estudiantes (50\% aproximadamente) lograron movilizarse del nivel bajo $(100 \%)$ a los niveles medio y alto $(40 \%$ y $11 \%$, en el caso del grupo 1 y $39 \%$ y $11 \%$ en el caso del grupo 2, respectivamente). Estos resultados evidencian que los estudiantes de ambos grupos lograron no solo reconocer la estructura de las crónicas, sino también producir un texto que atendiera en términos generales a dicha estructura. 
La secuencia didáctica, de enfoque comunicativo, como propuesta pertinente para la enseñanza del lenguaje y la formación docente

En las gráficas 13 y 14 se presentan los resultados para la dimensión lingüística textual.

\section{Gráfica 13. Comparativo Lingüística Textual Grupo 1}

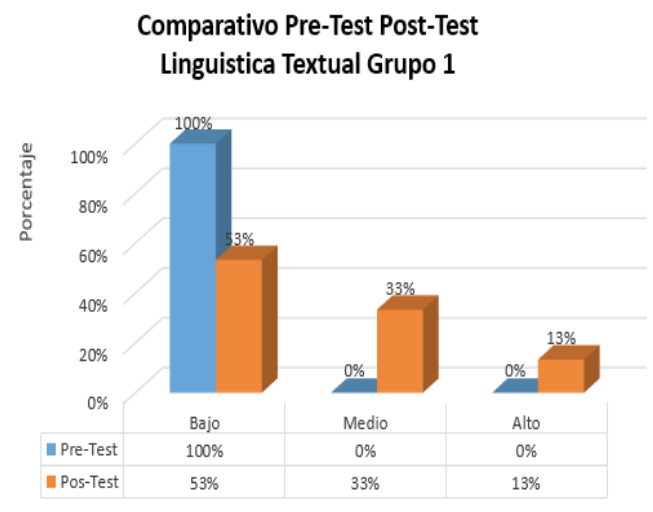

Gráfica 13. Comparativo Lingüística Textual Grupo 2

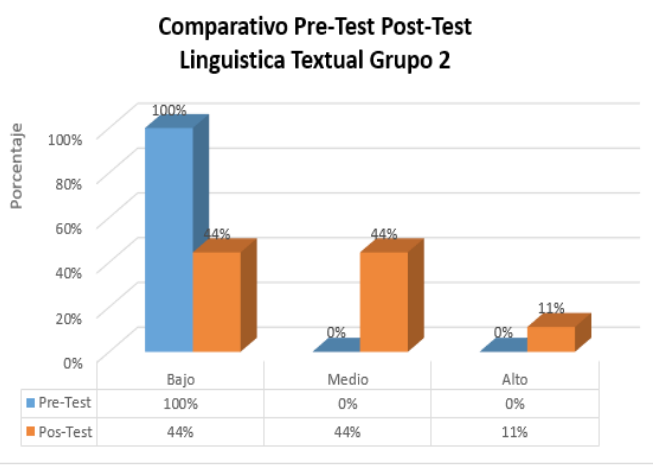

De acuerdo a las gráficas 13 y 14 , se observa que ambos grupos experimentaron el mismo comportamiento en relación a la dimensión lingüística textual, lo cual se evidencia en una progresión positiva en cuanto al desempeño de los estudiantes que se movilizaron desde el nivel bajo $(100 \%)$ hacia los niveles medio $(33 \%$ para el grupo 1 y $44 \%$ para el grupo 2) y alto ( $13 \%$ y $11 \%$ respectivamente); es decir, el grupo 2 registró unos avances levemente superiores a los estudiantes del grupo 1.

En la ilustración 1 se muestran evidencias del desarrollo de los talleres \# 2 y \#3, orientados al trabajo con la Situación de Comunicación.

Ilustración 1. Evidencias del trabajo de los estudiantes en los talleres \# 2 y \#3

Taller \# 1

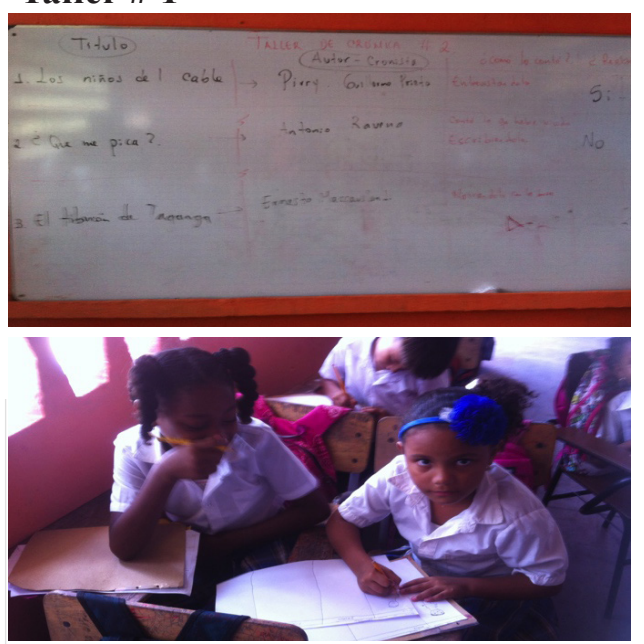

Taller \# 2

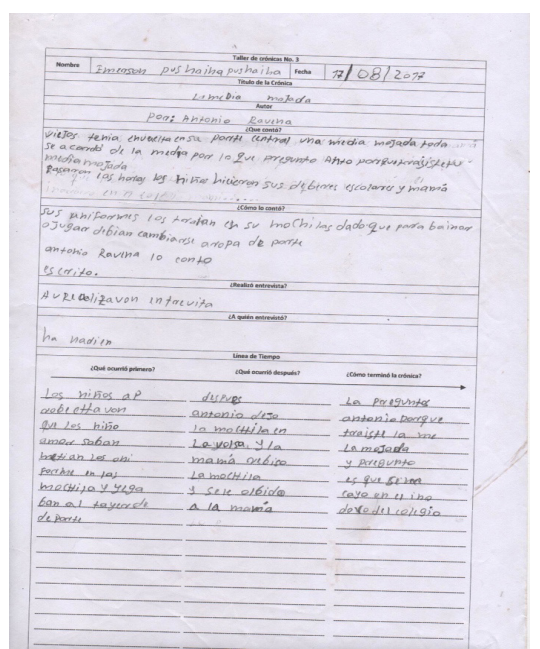

Revista de Investigación 
En las ilustraciones 2 y 3 se pueden observar las producciones del mismo estudiante del grupo 1 durante el PreTest y el Pos-Test, mientras que en la No. 2 se muestra la comparación de las producciones de un estudiante del grupo 2

Ilustración 2. Pre-Test y Pos-Test realizado por la estudiante $N .^{\circ} 6$ del grupo 1

\section{Pre-Test}

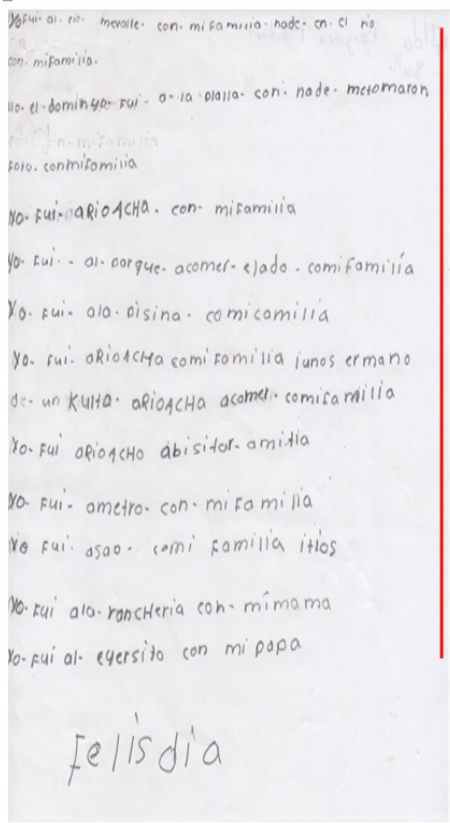

Rojo: Contenido

Amarillo: Enunciador

Azul: Destinatario

\section{Pos-Test}

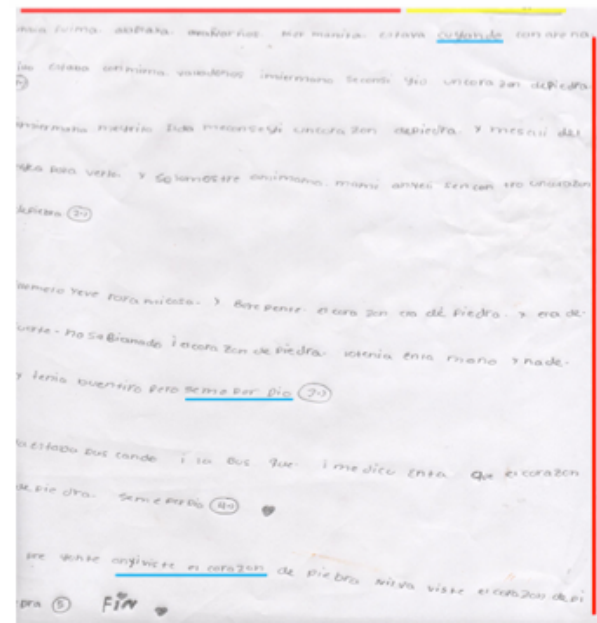

Ilustración 3. Pre-Test y Pos-Test realizado por la estudiante $\mathrm{N} \cdot{ }^{\circ} 7$ del grupo 2

\section{Pre-Test}

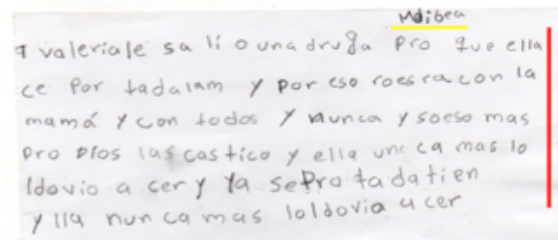

Rojo: Contenido

Amarillo: Enunciador

Azul: Destinatario

\section{Pos-Test}

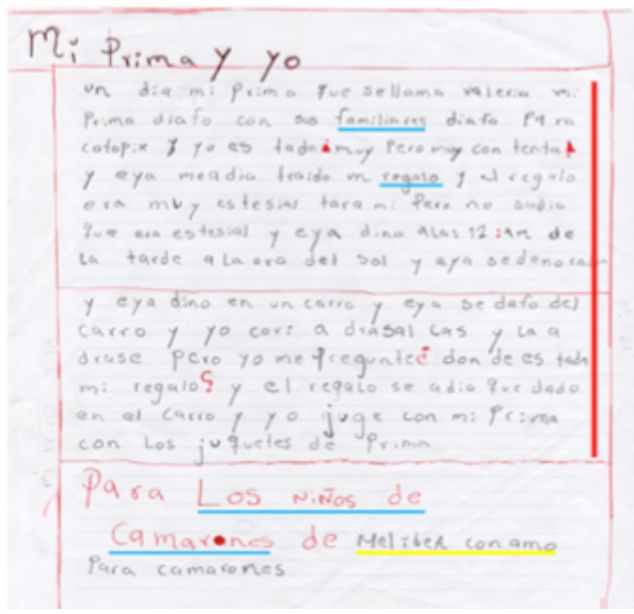


La secuencia didáctica, de enfoque comunicativo, como propuesta pertinente para la enseñanza del lenguaje y la formación docente

En los siguientes ejemplos se puede observar las evidencias del trabajo de los estudiantes en torno a los aspectos superestructurales del texto en contexto:

Ilustración 4. Evidencias del desarrollo de actividades de trabajo en torno a la Superestructura

\section{Pre-Test}

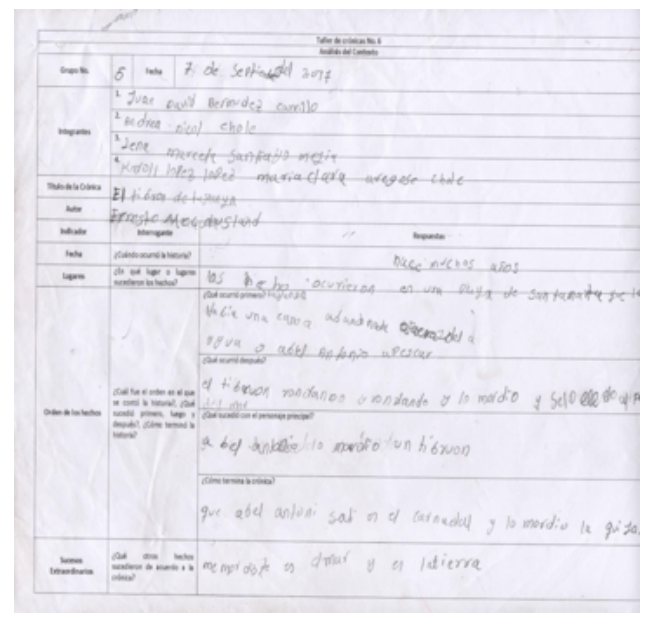

\section{Pos-Test}

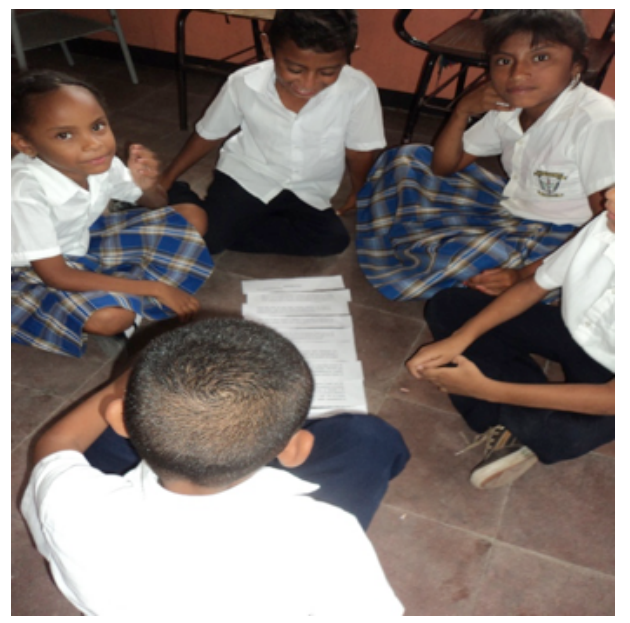

Por su parte, las ilustraciones No. 5 y No. 6 muestran las producciones de los mismos estudiantes durante el Pre-Test y el Pos-Test, del grupo 1 y del grupo 2 respectivamente.

Ilustración 5. Pre-Test y Pos-Test realizado por la estudiante $N .^{\circ} 1$ del grupo 1

\section{Pre-Test}

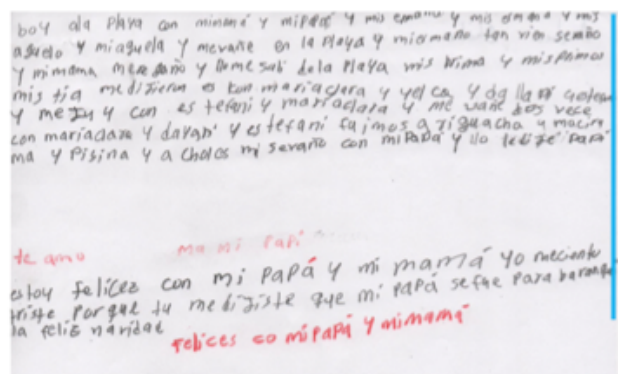

Rojo: Título

Azul: Presentación

Amarillo: Desarrollo Cronológico

Verde: Cierre

\section{Pos-Test}

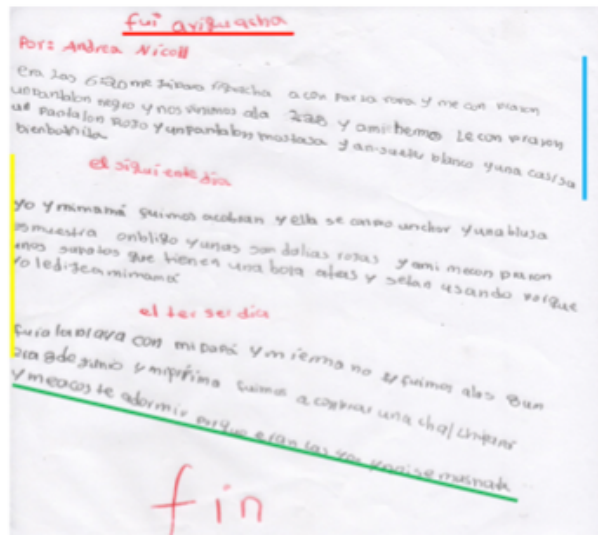

A continuación, en las ilustraciones 8 y 9 se observan las principales transformaciones en dos escritos, desde la lingüística textual. 


\section{Ilustración 8. Pre-Test y Pos-Test realizado por la estudiante $N .^{\circ} 2$ del grupo 1}

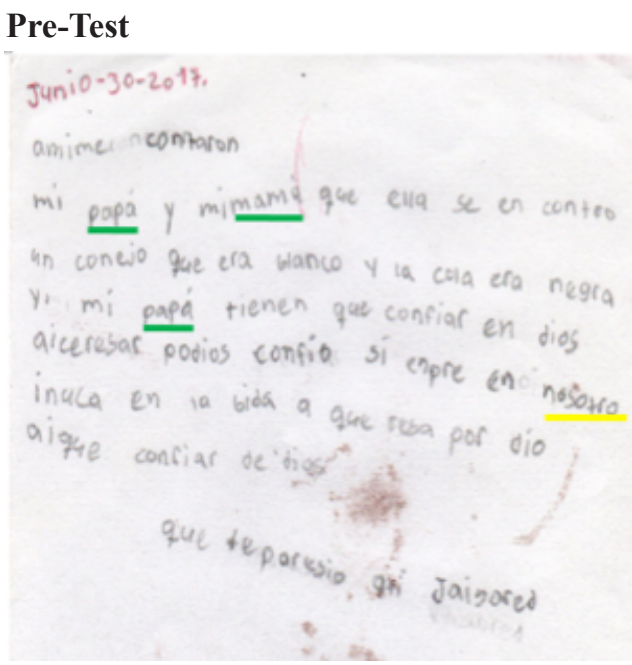

Rojo: Modalizaciones

Azul: Progresión temática

Amarillo: Anáforas y sustitutos

Verde: Puntuación

\section{Pos-Test}

\section{el primer dia quelo conosi a ra nena.}

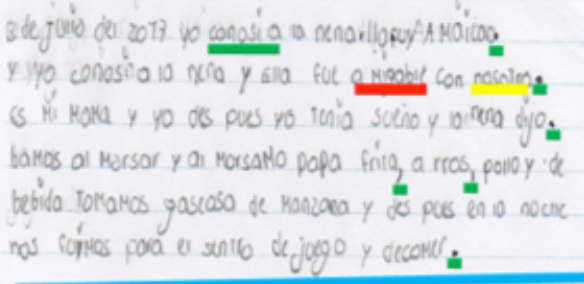

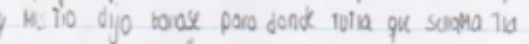
mosia y avo dorm

\section{Para los niño de cararono}

con arbor jásarts.

\section{Análisis cualitativo de las prácticas de enseñanza}

El complemento cualitativo contempló los resultados del análisis de las prácticas de los dos maestros investigadores, a partir de la información recogida en los diarios de campo durante la implementación de laS.D. ${ }^{4}$ Cabe resaltar que la S.D. se desarrolló a traves de 19 sesiones, las cuales comprendieron un total de 36 periodos de clase para el grupo 1 y de 40 periodos de clase para el grupo 2.

El análisis se organiza en las tres fases de la S.D.: preparación, desarrollo y evaluación.

\section{Profesor 1}

\section{- Fase de preparación}

En esta fase primaron las categorías de Descripción, Autopercepción y Autoevaluación; pues inicialmente enfoqué la mayor parte de mi interés en relacionar las características físicas del aula de clases y de los fenómenos generados en ella, sumado a la descripción de los sentimientos o ideas sobre mis actos durante las jornadas de trabajo.

\section{- $\quad$ Fase de desarrollo}

Durante la fase de desarrollo de la S.D. la reflexión y el análisis de mi práctica docente se enfocó mayoritariamente en la Autopercepción, es decir, en

\footnotetext{
4 La redacción de este apartado se realiza en primera persona, con la finalidad de ser coherentes con el texto que se produce y poder dar cuenta de las reflexiones realizadas por los docentes de manera personal.
} 
La secuencia didáctica, de enfoque comunicativo, como propuesta pertinente para la enseñanza del lenguaje y la

formación docente

descripción de mis sentimientos o ideas sobre mis actuaciones, destacando los sentimientos negativos (de impaciencia, ansiedad y miedos) y positivos (satisfacción, sorpresa, tranquilidad $\mathrm{y}$ orgullo) ante las actividades a desarrolladas mediante el proyecto. Además se presentó la categoría de Percepción de los estudiantes, atendiendo al interés por sus reacciones e impresiones en las actividades propuestas. "Siento satisfacción personal al escuchar las narraciones orales de manera detallada, fluida y con confianza por parte de los estudiantes de las crónicas abordadas en las sesiones de trabajo anteriores". (Sesión 11, clase 1).

\section{- Fase de cierre}

Esta fase constituyó el periodo más impactante del proyecto, atendiendo a los notables cambios en la disposición de trabajo de los estudiantes, las evidencias de aprendizaje, comprensión $\mathrm{y}$ manifestaciones de saberes en torno a los textos analizados y las producciones textuales desarrolladas. En este caso el énfasis en el diario de campo se mantuvo en mis impresiones sobre los estudiantes (Percepción de los estudiantes), especialmente en el marcado cambio de interés inicial por una nota sumativa, "-Profe califíqueme mi crónica, póngame la calificación" (Sesión 2, clase 2).

\section{Profesor 2}

\section{- Fase de Preparación}

Esta fase de preparación, la desarrollé en una sesión a través de dos clases, la cual orienté en mayor medida a la
Autopercepción, teniendo en cuenta que generó muchos sentimientos encontrados, por ejemplo, el saber que estaría trabajando en una serie de actividades muy bien articuladas y pensadas, destinadas a cumplir con un objetivo particular y a las cuales tendría que ceñirme de manera fiel, me hacían sentir nerviosa, expectante, entusiasmada y prevenida.

\section{- $\quad$ Fase de desarrollo}

Conociendo el grado de responsabilidad por la consecución de los objetivos de las diferentes sesiones de trabajo y el compromiso establecido con mis estudiantes, inicié esta fase con muchas expectativas, concentrada en cada una de las actividades y dispuesta a dar lo mejor, pues mis estudiantes habían respondido muy bien a la primera fase y eso me motivaba a esforzarme tanto personal como académicamente (Autopercepción).

\section{- Fase de cierre}

Esta fase fue la que más me gustó, me sentía satisfecha de los logros alcanzados por parte de mis estudiantes, lo dispuestos que estaban a terminar con las 19 sesiones de trabajo para cumplir con la publicación de libro y leer las crónicas de los compañeritos de la otra institución, como un intercambio de lectores y escritores (Percepción de los estudiantes). Me sentí muy orgullosa porque al final, ellos mismos estaban en capacidad de hacer revisiones, correcciones y evaluaciones en sus escritos (Autopercepción). 


\section{Discusión}

De acuerdo a los resultados de la investigación, respecto a los desempeños iniciales de los estudiantes, se presentaron dificultades en el proceso de producción relacionadas basicamente con tres aspectos: El primero vinculado a la aproximación al texto, derivado posiblemente de la creencia que aún no podían escribir textos completos porque no tenían el código alfabético, es decir "no sabían leer", y por tanto debían hacer ejercicios de escritura y lectura sin sentido para ellos; esta situaciòn a su vez, generaba que se viera el lenguaje escrito como una tarea que se enfrentaba con apatía (Castaño, 2014). El segundo, relacionado con la concepción de escritura como producto terminado, por lo cual desarrollaban una sola versión que era considerada como la definitiva, y por tanto no requería de planeación, revisión $\mathrm{y}$ reescritura, tantas veces como fuera necesario. Finalmente, el no creer que estaban en capacidad de ser escritores, pues ni ellos ni sus profesores creían en dicha potencialidad.

Este conjunto de dificultades posiblemente obedece a prácticas de enseñanza del lenguaje, en las cuales se privilegia su tratamiento normativo, la exposición a un solo tipo de textos (narrativos: cuentos) con un abordaje centrado en una estructura de inicio, nudo y desenlace, la descripción de hechos y personajes que concluyen en la elaboración de un dibujo para saber qué comprendieron, desde el supuesto que se están atendiento a las necesidades de los niños: dibujar (Sánchez, 2014).
Frente a estas dificultades, reconocidas incluso antes de iniciar la planeación de la secuencia, se hizo una propuesta centrada en el uso del lenguaje en situaciones reales de comunicación (Teberosky,1990 y Tolchinsky, 1993). Este primer aspecto, también generó cambios en la manera de pensar la escritura: los textos deberían ser comprendidos por otros. La secuencia partió de reconocer y valorar el contexto de los estudiantes, trajo este contexto y sus viviencias como pretexto y medio para la escritura con sentido.

Luego puso en juego varios supuestos, el primero de ellos era que los niños pueden comprender textos aun cuando no dominen el código alfabético, pues están en capacidad de hacer inferencias sobre lo que leen y escuchan. El segundo, fue poner en juego la lectura y la oralidad, desde el supuesto que se lee para escribir y hablar, por tanto, la secuencia le dio la palabra a los estudiantes, posibilitó la expresión de pensamientos y sentimientos frente a los hechos cotidianos que les ocurren a ellos mismos o que ocurren en sus cuadras, barrios, rancherías. El tercero, es la democratización y por tanto el respeto mutuo en el aula, todos eran estudiantes aprendiendo, hablando, haciendo "algarabía", en un aula viva, en la que todos podrían hablar, sin que importara la etnia o el color. El cuarto, es que la escritura tiene diversas funciones que van desde entretener hasta solicitar, por ejemplo, la elaboración de la carta para invitar al experto, la elaboración de preguntas para este, y la escritura misma de la crónica. El quinto, pasar de la concepción de escritura como producto a la escritura como proceso, $\mathrm{y}$ 
La secuencia didáctica, de enfoque comunicativo, como propuesta pertinente para la enseñanza del lenguaje y la formación docente

por ello la necesidad de volver al texto las veces que fuese necesario, hasta que se sintieran satisfechos con el producto. El sexto, que todos eran aprendices y que podrían ayudarse mutuamente, en un trabajo colaborativo, en el que se evaluaban mutuamente con el único ánimo de mejorar la escritura. Finalmente y quizas el más importante, pensarse como escritores, como productores de sus propios textos y por tanto con una voz que trascendería los límites del aula de clase.

\section{Conclusiones}

A partir de la contrastación de los resultados del Pre-Test y Pos-Test y el análisis el estadístico de la prueba T-student, se rechaza la Hipótesis Nula y se acepta la Hipótesis de Trabajo, por tanto se puede afirmar que la implementación de una S.D. de enfoque comunicativo, mejoró significativamente la producción de textos narrativos, tipo crónicas, de los estudiantes del grado $2^{\circ}$ de los dos grupos de estudio. Estos resultados evidencian la potencialidad de una secuencia didáctica que parte de reconocer a los estudiantes y el contexto en el que viven, les da la palabra para que desde su propia visión relaten lo que ven y sus sentimientos al respecto, y cree en su potencialidad para irse pensando y construyendo como escritores. Una secuencia didáctica que se desmarca del acento en la codificación y revisión de aspectos como la ortografía o la segmentación, para centrarse en la lectura y la escritura situada personal y socialmente, con propósitos comunicativos construidos de manera colaborativa.
Después del desarrollo de la secuencia didáctica se evidenció un avance significativo en los procesos de producción escrita, en las tres dimensiones propuestas. Específicamente en la dimensión situación de comunicación, lograron posicionarse como cronistas, capaces de narrar y expresar su posición frente a los hechos narrados para un potencial lector.

Respecto a la superestructura, los estudiantes comprendieron que un texto tiene una manera de ser contado, atendiendo a los requerimientos formales en los que se inscribe, y tambièn al desafío que implica hacerlo para un posible lector que puede no conocer la realidad en la que está posicionado el escritor. En este marco los estudiantes repensaron el título y su sentido, hicieron un párrafo de presentación del contexto y personajes de la crónica seguido de un segmento de narración cronológica del hecho o conjunto de acciones como eje central de la historia y finalizando con un cierre coherente con esta.

Y finalmente, en relación a la lingüística textual, entendieron el sentido de utilizar distintos recursos lingüísticos como anáforas y sustitutos, modalizadores y signos de puntuación, para darle sentido e impronta a un texto en el que la subjetividad de quien cuenta es central.

Respecto a las prácticas de enseñanza de los dos maestros, puede plantearse que iniciaron una transformación de sus concepciones de enseñanza del lenguaje escrito, las cuales los llevaron 
a comprender la escritura como proceso que supera el énfasis evaluativo, basado en la escritura como tarea y el docente como destinatario único de las producciones de los estudiantes, para pensarlo desde una perspectiva interactiva y comunicativa, acorde con la naturaleza sociocultural del lenguaje.

Finalmente, la reflexión sobre las propias prácticas de los docentes antes, durante y después de la implementación del proyecto de investigación permite ratificar que sí es posible que los educadores analicen lo que sucede en las aulas para confrontan sus prácticas pedagógicas, salir de la zona de confort, y asumir posiciones críticas que los llevan a transformar lo que hacen, en beneficio de los cientos de niños que pasan por sus aulas.

\section{Referencias bibliográficas}

Aguirre, P., y Quintero, Y. (2014). Incidencia de una secuencia didáctica desde una perspectiva discursivainteractiva en la producción de textos narrativos de estudiantes de grado $1^{\circ}$ de EBP, de la institución educativa la inmaculada de la ciudad de Pereira. (Tesis de maestría). Universidad Tecnológica de Pereira, Pereira. Recuperada de: http:// repositorio.utp.edu.co/dspace/bitstream/ handle/11059/4627/37241A284i. pdf? sequence $=3$

Barthes, R. (1985). Análisis estructural de los relatos Cuarta Edición. México: Premiá.

Bautista, A., y Cortés, J. (1998). Maestros Generadores de textos. Colombia: Escuela de Estudios Literarios de la Universidad del Valle.
Camps, A., Cotteron, J., Dolz, J., Farrera, N., Fort, R., Guasch, O., Martinez, A., Milian, M., Ribas, T., Rodríguez, C., Santamaria, J., Utset, M., Vila, M., y Zayas, F. (2003). Secuencias didácticas para aprender a escribir. España: Departamento de Didáctica de la lengua y la literatura. Universidad Autónoma de Barcelona.

Caro, M., Uribe, G., y Camargo, Z. (2009). Didáctica de la comprensión y producción de textos académicos. Armenia, Colombia: Universidad del Quindío.

Cassany, D. (1999). Los enfoques comunicativos: elogio y crítica. Lingüística y Literatura, 36 (37), 11-33.

Castaño, A. (2014). Prácticas de escritura en el aula: orientaciones didácticas para docentes. Bogotá, Colombia: Ministerio de Educación Nacional de Colombia.

Chomsky, C. (1957). Reading and phonolofy. Harvard Educational Review, 40 (3), 287-309.

Ferreiro, E. (1983). Psicogénesis de la Escritura. En C. Coll. (Ed.), Psicología Genética y aprendizajes escolares. Recopilación de textos sobre las aplicaciones pedagógicas de las teorías de Piaget (pp.79-90). Barcelona, España: Siglo XXI de España editores S.A.

Hayes, L., y Flower, J. (1980). The dynamics of composing: making plans and juggling constraints. Cognitive processes in writining. New Jersey, EE.UU: Lawrence Earlbaum Asociantes.

Hernández, R., Fernández, C., y Baptista, P. (2010). Metodología de la Investigación. México D.F., México: Editorial McGraHill/Interamericana Editores S.A.

Hymes, H. D. (1972). El enfoque comunicativo de la enseñanza de la lengua. Barcelona, España: Editorial Paidós Ibérica. 
Imbernón, F. (2012). La investigación educativa como herramienta de formación del profesorado. Revista Electrónica de Investigación Educativa REDIE, 14(2), $1-9$.

Instituto Colombiano para la Evaluación de la Educación-ICFES-. (2017). Resultados

Jolibert, J. (2002). Formar niños lectores/ productores de textos: Propuesta de una problemática didáctica integrada. Paris, Francia: Editorial Dolmen Ediciones.

Jolibert, J. y Sraïki, C. (2009). Niños que construyen su poder de leer y escribir. Recuperado de: https://docplayer. es/12523221-Ninos-que-construyen-supoder-de-leer-y-escribir.html

Lerner, D. (2001). Leer y escribir en la escuela: lo real, lo imposible y lo necesario. Barcelona, España: Editorial Fondo de cultura económica de España.

Ministerio de Educación Nacional de Colombia -MEN-. (2006). Estándares básicos de competencia del lenguaje. Formar en lenguaje: aperturas de caminos para la interlocución. Bogotá, Colombia: Ministerio de Educación Nacional.

Morales, M., y Arenas, E. (2017). Relatos, sueños y arte de mi pueblo: Produciendo cronicas con estudiantes de la I.E nuestra señora de la presentacion. (Tesis de maestría). Universidad Tecnológica de Pereira, Pereira. Recuperada de: http:// repositorio.utp.edu.co/dspace/bitstream/ handle/11059/7822/372623 M828. pdf? sequence $=1$.

Organización de las Naciones Unidas para la Educación, la Ciencia y la Cultura UNESCO. (1997). Documento 5: Tendencias en la Enseñanza del Lenguaje. Laboratorio Latinoamericano de Evaluación de la calidad de la educación. Recuperado de http://unesdoc.unesco.org/ images/0018/001836/183654s.pdf el 10 de 04 de 2016.
Ortiz, E. (2010). La crónica: lo que es y lo que no es. México D.F., México: Editorial Universidad Autónoma de México.

Pérez, M., y Rincón, G. (2009). Actividad, Secuencia Didáctica y Pedagogía por Proyectos: Tres Alternativas para la Organización del trabajo Didáctico en el campo del lenguaje. Recuperado de: https://scf563291e8cb4ead.jimcontent. $\mathrm{com} / \mathrm{download} / \mathrm{version} / 1434142807 /$ $\mathrm{module} / 8404496383 / \mathrm{name} /$ Alternativas \%20RED $\% 20 \mathrm{DE} \% 2 \overline{0}$ LENGUAJE.pdf

Pérez, T., Camacho, S., y Rodríguez, O. (2013). Las ciencias naturales y las prácticas pedagógicas en la formación del derecho al ambiente sano. (Tesis de maestría). Universidad Santo Tomás. Bogotá. Recuperada de: http://repository. usta.edu.co/bitstream/handle/11634/2569/ Montalvocastroalberto\% 20 . pdf? sequence $=1$ \&isAllowed $=y$

Perrenoud, P. (2011). Desarrollar una práctica reflexiva en el oficio de enseñar. Profesionalización y razón pedagógica. Colección Crítica y fundamentos, serie Formación y desarrollo profesional del profesorado. México D.F., México: Editorial GRAÓ.

Salcedo, A. (2012). La crónica: el rostro humano de la noticia. Pasos esenciales del género, desde la selección del tema hasta la escritura final. Recuperado de: http://bicentenario.fnpi.org/meteriales/la cronica_el_rostro_humano_de_la_noticia. pdf

Sánchez, C. (2014). Prácticas de lectura en el aula: orientaciones didácticas para docentes. Recuperado de: http:// www.colombiaaprende.edu.co/html/ micrositios/1752/articles-341024 recurso_1.pdf 
Scardamalia, M., y Bereiter, C. (1992).

Dos modelos explicativos de los procesos de composición escrita. Infancia y Aprendizaje: Journal for the Study of Education and Development, 210 (58), 4364.

Schön, D. (1987). La formación de profesionales reflexivos. Hacia un nuevo diseño de la enseñanza y el aprendizaje en las profesiones. Barcelona, España: Editorial Paidós Ibérica.

Teberosky, A. (1990). El lenguaje escrito y la alfabetización. Lectura y Vida, 11 (12), $1-15$.

Tolchinsky, L. (1993). Aprendizaje del Lenguaje Escrito: escribir en la escuela. Barcelona, España: Editorial Anthropos.

Zuluaga, O. (1984). El maestro y el saber pedagógico en Colombia, 1821-1948. Medellín, Colombia: Editorial Magisterio. 